\title{
Mammographic Image Enhancement Based on SWT and High Boost Filtering
}

\author{
Arya Devi P. S. and M. G. Mini
}

\begin{abstract}
A Mammogram is a competent tool in early detection of breast cancer. Image enhancement in mammographic images increases the diagnosability of abnormalities in the images. The classical image enhancement techniques are not adept to improve the diagnostic features in mammograms. The enhancement technique proposed in this paper utilizes Stationary Wavelet Transform (SWT), modulus maxima and high boost filtering. The image is decomposed using SWT and its modulus maximum is determined. A fraction of the high pass filtered image obtained as the result of SWT decomposition and modulus maxima is added to original image. The scheme is evaluated visually and objectively using measures like contrast, PSNR etc. The performance measures are evaluated for different category of images and found to be suitable to all categories of mammographic images.
\end{abstract}

Index Terms-Contrast improvement index, EMEE, mammogram, PSNR, stationary wavelet transform.

\section{INTRODUCTION}

Breast cancer alone is expected to account for $29 \%$ of all new cancers among women [1]. A mammogram is the most effective technique for breast cancer screening and early detection of masses or abnormalities; it can detect 85 to 90 per cent of all breast cancers [2]. The early signs of breast cancer are masses, calcifications, architectural distortion and bilateral asymmetry [3]. The abnormalities like masses and micro calcifications are hard to detect since they have low contrast compared to contiguous breast tissues. Image enhancement can improve the radiologists' perception to subtle and more accurate diagnosis [4].

Image enhancement includes techniques such as contrast and intensity manipulation, noise reduction, background removal, edges sharpening and filtering. The usual task of mammogram enhancement is to increase the contrast between regions of interest (ROI) and background and to sharpen the edges or borders of regions of interest [5]. However, some image enhancement techniques may distort diagnostic features, appearance and shape, leading to wrong diagnosis [6]. The main problem is the under-enhancement of some regions and over-enhancement of others. Under-enhancement can cause false negatives, while

Manuscript received May 14, 2014; revised August 5, 2014. This work was supported by Engineering and Technology Programs, Kerala State Council for Science, Technology and Environment (KSCSTE), Thiruvananthapuram.

The authors are with the Department of Electronics, Model Engineering College, Kochi, Kerala, India (e-mail: aryaps@mec.ac.in, mininair@mec.ac.in). over-enhancement can cause false positives [5].

The methods used to manipulate mammogram images can be categorized into four main categories; the conventional enhancement techniques, the region-based enhancement techniques, the feature-based enhancement techniques, and the fuzzy enhancement techniques. Conventional enhancing techniques are fixed neighbourhood techniques that modify images based on global properties. Region-based methods for enhancing the contrast of mammogram features are based on the surroundings i.e., local properties. The feature based enhancement methods are those methods that utilises wavelet domain enhancement and the fuzzy enhancement techniques are methods that apply fuzzy operators and properties to enhance mammogram features [2].

Sivaramakrishna et al. compared the performance of several contrast enhancement algorithms: adaptive unsharp masking, contrast-limited adaptive histogram equalization, adaptive neighbourhood contrast enhancement, and wavelet based enhancement in a preference study. In a majority of the cases with micro-calcifications, the adaptive neighbourhood contrast enhancement algorithm provided the most-preferred images $(58 \%)$, followed by the unsharp masking algorithm. Feature based enhancement methods can be used to enhance both masses and micro-calcifications [7]. Chang and Laine suggested an enhancement algorithm based on over-complete multi-scale wavelet analysis [8]. Another method proposed by Gagnon et al., puts forward a simple multi-scale sharpening enhancement algorithm based on the hidden zero-crossing property of the complex symmetric Daubechies wavelets [9]. A. Papadopoulosa, D. I. Fotiadisb and L. Costaridouc found that local range modification and wavelet-based linear stretching suited for enhancement of images with micro-calcifications [10]. Scharcanski and Jung described an approach for noise suppression and enhancement of mammogram images and that can be effective in screening dense regions of the mammograms [11]. In this paper we propose a method to enhance mammographic images using Stationary wavelet transform (SWT), wavelet modulus maxima of the transform and unsharp masking. The paper is organized as follows: related work and background, proposed method, performance measures, results $\&$ discussion and conclusion.

\section{RELATED WORK AND BACK GROUND}

The aim of image enhancement is to improve the interpretability or perception of information in images for human viewers, or to provide better input for other automated image processing techniques. The enhancement method developed is based on SWT, wavelet modulus maxima and 
high boost filtering.

The basic idea of stationary wavelet transform is to fill the gap caused by decimation in the standard wavelet transform resulting in over determined representation of the original data, having much statistical potential [12]. The SWT is an inherently redundant scheme as the output of each level of SWT contains the same number of samples as the input. So for a decomposition of $N$ levels, there is a redundancy of $N$ in the wavelet coefficient. SWT is similar to the DWT except that the filters are up-sampled, instead of sub-sampling the signal at each level of decomposition. Each level's filters are up-sampled versions of the previous ones. The decomposition and filters are shown in Fig. 1 [13].

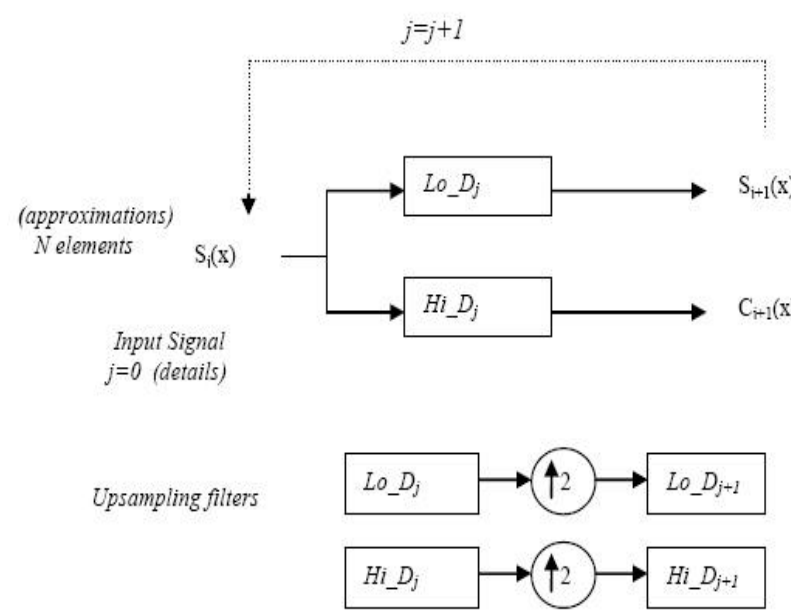

Fig. 1. SWT decomposition and filters.

The modulus maxima of the wavelet transform provide a nearly complete characterization of an image. A definition of local maxima of the wavelet transform modulus is: Let $W f(s$, $x$ ) be the wavelet transform of a function $f(x)$. The modulus maximum is any point $\left(s_{0}, x_{0}\right)$,such that $\left|W f\left(s_{0}, x\right)\right|<\left|W f\left(s_{0}, x_{0}\right)\right|$ when $x$ belongs to either a right or the left neighbourhood of $x_{0}$, and $\left|W f\left(s_{0}, x\right)\right|<=\left|W f\left(s_{0}, x_{0}\right)\right|$ when $x$ belongs to the other side of the neighbourhood of $x_{0}$. The local maxima of the wavelet transform modulus provide enough information to detect and analyze all discontinuities inside images[14]. Consider wavelet decomposition of an image $f(x, y)$ at scale $j$, we get an approximation and three detail images represented as $W_{j}^{h} f, W_{j}^{v} f$ and $W_{j}^{d} f$, where the superscripts $h, v$ and $d$ denote the horizontal, vertical and diagonal details. Mallat and Zhong characterize the image edges at scale $j$ by the local modulus maxima denoted as $M_{j} f$

$$
M_{j} f(x, y)=\sqrt{\left|W_{j}^{h} f(x, y)\right|^{2}+\left|W_{j}^{v} f(x, y)\right|^{2}}
$$

In the unsharp masking approach for image enhancement, a fraction of the high-pass filtered image is added to the original image to form the enhanced image [15]. High boost filtering is a type of unsharp masking. The high boost filter not only preserves the low frequency information but also enhances the high frequency detail information. This enhances the similarity feature value within similar regions and dissimilarity feature value among the dissimilar regions. The high boost filter is simple and implementation cost is less [16].

\section{The PROPOSED METHOD}

The image is cropped to a size of $256 \times 256$. SWT is performed on the cropped image up to 3 levels. For each level, the absolute maximum wavelet coefficient, called wavelet modulus maximum, is computed. Using absolute maximum of the wavelet modulus and an experimentally determined threshold $T_{n}$, only some of detail coefficients are retained while the rest is discarded. The high pass image obtained as a result is used for high boost filtering. $T_{n}$ is set up to include all wavelet coefficients whose absolute value is within the range of the threshold of the wavelet modulus maximum. Let $W f\left(2,{ }^{i} x_{0}, y_{0}\right)$ be wavelet modulus of the image at the level $i$ which has highest absolute value $M_{i}$ among all the coefficients of each scale. Any wavelet coefficients $W f\left(2^{i}\right.$, $x, y)$ at each scale satisfying either of the following:

$$
\begin{aligned}
& \text { (a) } W f\left(2^{i}, x, y\right) \leq-M_{i}+T_{n} \text { if } W f\left(2^{i}, x, y\right)<0 \\
& \text { (b) } W f\left(2^{i}, x, y\right) \geq M_{i}-T_{n} \text { if } W f\left(2^{i}, x, y\right) \geq 0
\end{aligned}
$$

are kept unchanged, whereas all the other wavelet coefficients are set to zero before the reconstruction of the image. The approximation is also put to zero and the inverse SWT is calculated. The original image is enhanced by adding a fraction of the reconstructed image to the original.

\section{EVALUATION CRITERIA}

There is no general rule for determining quality of image enhancement when it comes to human perception. However, when image enhancement techniques are used as pre-processing tools for other image processing techniques, quantitative measures can determine which techniques are most appropriate. We can verify enhancement of an image by visual inspection. The objective measures used here for measuring enhancement are contrast, measure of enhancement, entropy, contrast improvement index and PSNR. The contrast of an image is evaluated by employing the metric function given:

$$
C_{C}=\frac{1}{M N} \sum_{i=1}^{M} \sum_{j=1}^{N} f^{\prime 2}(i, j)-\left|\frac{1}{M N} f^{\prime}(i, j)\right|^{2}
$$

where $M$ and $N$ are height and width of the image, respectively, and $f^{\prime}(i, j)$ is the enhanced image. The larger the value of $C_{C}$, the better the contrast of the image. A quantization measure of contrast enhancement defined by a contrast improvement index (CII), is expressed as

$$
\mathrm{CII}=\frac{C_{\text {processed }}}{C_{\text {original }}}
$$

where $C_{\text {processed }}$ and $C_{\text {original }}$ are the contrasts of the processed and original images, respectively. $C$ is the average value of the local region contrast in the processed or original image. Thus, the CII value of original image is equal to one. The local contrast at each pixel is measured as $\left(X_{\max }-\right.$ $\left.X_{\min }\right) /\left(X_{\max }+X_{\min }\right)$ in its local window size [17]. The 
measurement of enhancement or measure of improvement (EME) is another performance measure used in this work. For defining enhancement measure by entropy (EMEE), consider an image $x(n, m)$ be split into $k_{1} k_{2}$ blocks $w_{k l}(i, j)$ of sizes $l_{1} \times l_{2}$. EMEE is given as

$$
E M E E=\max _{\phi \in\{\Phi\}} \chi(E M E(\phi))
$$

where $\chi(E M E(\Phi))$ is defined as

$$
\chi(E M E(\phi))=\left(\frac{1}{k_{1} k_{2}} \sum_{i=1}^{k_{1}} \sum_{j=1}^{k_{2}} \frac{I_{\mathrm{max} ; k, l}^{w}}{I_{\mathrm{min} ; k, l}^{w}} \log \frac{I_{\mathrm{max} ; k, l}^{w}}{I_{\mathrm{min} ; k, l}^{w}}\right)
$$
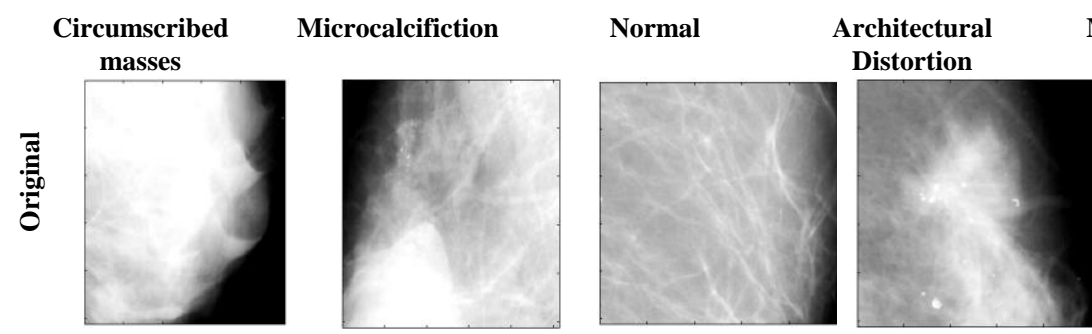

Miscellaneous

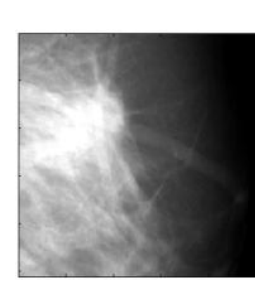

Spiculated mass
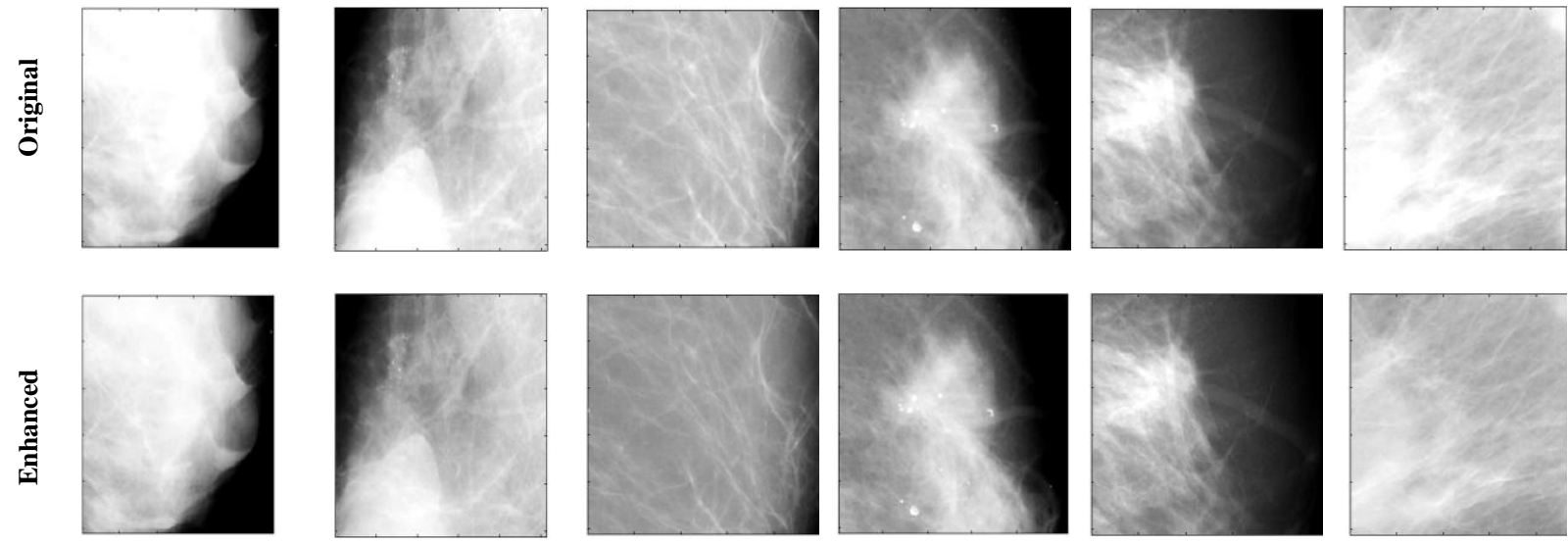

Fig. 2. Original and Enhanced versions of normal and different abnormal images.

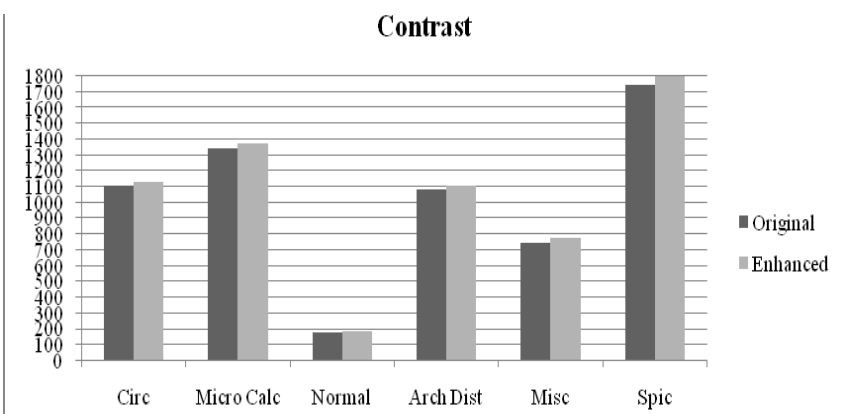

Fig. 3. Contrast comparison of original and enhanced images.

CII

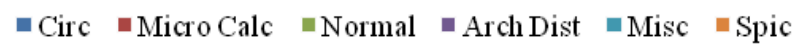

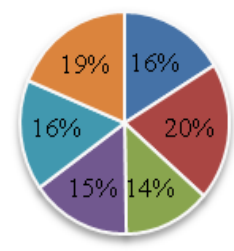

Fig. 5. Pie chart of CII.

\section{RESUlTS AND DisCUSSIONS}

The database used for this study is mini-MIAS database of mammograms which contains 322 images of size $1024 \times 1024$. It also includes radiologist's 'truth'-markings on the locations of any abnormalities that may be present [20].

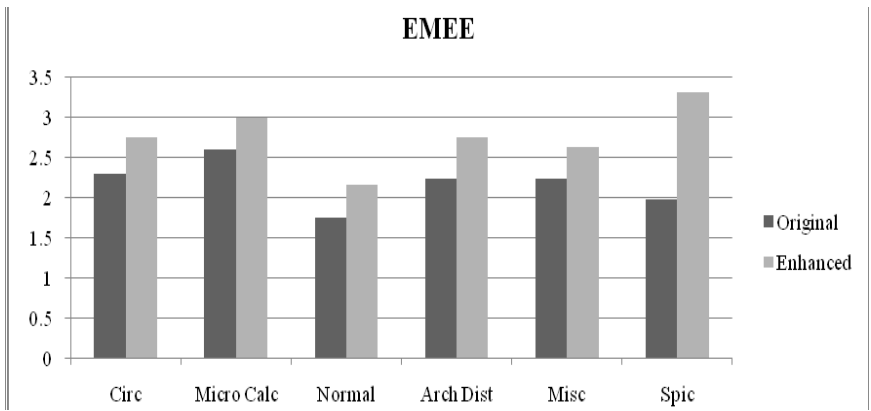

Fig. 4. Comparison of EMEE for original and enhanced images.

PSNR

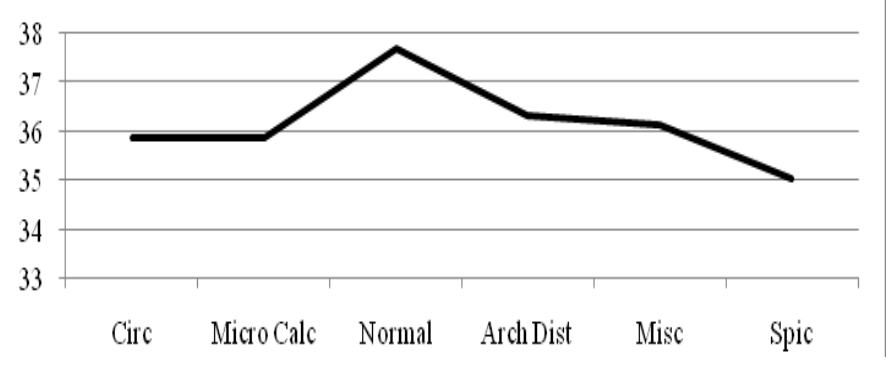

Fig. 6. PSNR comparison.

Normal images as well as images containing various types of abnormalities were enhanced using proposed method. The results of enhancement for various types of normal abnormal mammogram images are shown in Fig. 2.

The average value of performance measures for each type of image in the database is calculated and the results are 
tabulated in Table I which clearly show the improvement in image quality. The plots of contrast and EMEE against different types of images are shown in Fig. 3 and Fig. 4 respectively. From the pie chart of CII given in Fig. 5 we can see that there is not much difference in CII between different categories of images. The overall contribution to CII from normal images is found to be less than those images with abnormalities. Rajkumar K. K. and G. Raju [21] compared enhancement techniques based on wavelet and top hat filtering and bit plane wavelet decomposition methods in terms of average CII using same database. The comparison shown in Table II indicates that the proposed method offers a better CII. The PSNR values also indicate enhancement in the quality of images. The plot shown in Fig. 6 suggests that normal images show better PSNR.

TABLE I: COMPARISON OF PERFORMANCE MEASURES FOR DIFFERENT TYPES OF IMAGES

\begin{tabular}{|c|c|c|c|c|c|}
\hline \multirow[t]{2}{*}{ Type of image } & \multirow{2}{*}{$\begin{array}{l}\text { Original / } \\
\text { Enhanced }\end{array}$} & \multicolumn{4}{|c|}{ Performance Measures } \\
\hline & & Contrast & EMEE & CII & PSNR \\
\hline \multirow{2}{*}{$\begin{array}{l}\text { Circumscribed } \\
\text { Masses }\end{array}$} & Original & 1105.61 & 2.30 & \multirow{2}{*}{1.32} & \multirow{2}{*}{35.86} \\
\hline & Enhanced & 1122.72 & 2.75 & & \\
\hline \multirow[t]{2}{*}{ Microcalcification } & Original & 1335.62 & 2.59 & \multirow[b]{2}{*}{1.64} & \multirow[t]{2}{*}{35.88} \\
\hline & Enhanced & 1368.75 & 2.98 & & \\
\hline \multirow[t]{2}{*}{ Normal } & Original & 176.21 & 1.74 & \multirow{2}{*}{1.08} & \multirow[t]{2}{*}{37.68} \\
\hline & Enhanced & 181.59 & 2.15 & & \\
\hline \multirow{2}{*}{$\begin{array}{l}\text { Architectural } \\
\text { Distortion }\end{array}$} & Original & 1077.07 & 2.23 & \multirow{2}{*}{1.24} & \multirow[t]{2}{*}{36.31} \\
\hline & Enhanced & 1104.37 & 2.74 & & \\
\hline \multirow[t]{2}{*}{ Miscellaneous } & Original & 739.24 & 2.23 & \multirow[b]{2}{*}{1.32} & \multirow[t]{2}{*}{36.12} \\
\hline & Enhanced & 770.41 & 2.63 & & \\
\hline \multirow{2}{*}{$\begin{array}{l}\text { Spiculated } \\
\text { Masses }\end{array}$} & Original & 1740.12 & 1.97 & \multirow[b]{2}{*}{1.52} & \multirow[t]{2}{*}{35.03} \\
\hline & Enhanced & 1791.59 & 3.31 & & \\
\hline
\end{tabular}

TABLE II: COMPARISON OF CII IN DIFFERENT ENHANCEMENT METHODS

\begin{tabular}{ll}
\hline \hline Method & CII \\
\hline Top Hat & 1.027 \\
\hline
\end{tabular}

\begin{tabular}{lc} 
Wavelet decomposition (Sure Shrink) & 1.165 \\
\hline Top Hat +Sure Shrink & 1.242 \\
\hline Top Hat + level dependent Wavelet Shrink & 1.203 \\
\hline Top Hat +Visual Shrink & 1.186 \\
\hline Top Hat +level dependent Visual Shrink & 1.188 \\
\hline Top Hat + modified level independent Visual Shrink & 1.185 \\
\hline Top Hat +Bit Plane decomposition & 1.069 \\
\hline Proposed method & 1.353 \\
\hline \hline
\end{tabular}

\section{CONClusions}

In this method SWT and its modulus maxima is exploited to bring out the enhancement of mammographic images. The use of SWT and modulus maxima removes the noise present in the image and makes it visually appealing. The enhancement is demonstrated both subjectively and objectively using Contrast, EMEE, CII and PSNR. The increase in contrast is found to be less for normal images when compared with the ones having any sort of abnormality. We can conclude that this method may be used as a pre-processing step in Computer Aided Diagnosis (CAD) of breast cancer from mammographic images.

\section{REFERENCES}

[1] American Cancer Society, "Cancer statistics 2014: Death rates continue to drop," Science Daily, 7 January, 2014.

[2] M. Biltawi, N. Al-Najdawi, and S. Tedmori, "Mammogram enhancement and segmentation methods: Classification, analysis, and evaluation." in Proc. 13th International Arab Conference on Information Technology ACIT2012, December 10-13, pp. 477-485.

[3] J. Bozek, M. Mustra, K. Delac, and M. Grgic, "A Survey of image processing algorithms in digital mammography," Recent Advances in Multimedia Signal Processing and Communications, pp. 631-657, Springer Berlin Heidelberg, 2009.

[4] R. Rangayyan, F. Ayres, and J. Desautels, "A review of computer-aided diagnosis of breast cancer: Toward the detection of subtle signs," Journal of the Franklin Institute, vol. 344, pp. 312-348, Elsevier, 2007.

[5] H. Cheng, X. Cai, X. Chen, X. L. Hu, and X. Lou, "Computer-aided detection and classification of micro-calcifications in mammograms: A survey," Pattern Recognition, vol. 36, no. 12, pp. 2967-2991, 2003.

[6] C. Kimme-Smith, R. Gold, L. Bassett, L. Gormley, and C. Morioka, "Diagnosis of breast calcifications: comparison of contact, magnified, and television-enhanced images," American Journal of Roentgenology, vol. 153 , no. 5 , pp. 963-967, 1989.

[7] R. Sivaramakrishna, N. Obuchowski, W. Chilcote, G. Cardenosa, and K. Powell, "Comparing the performance of mammographic enhancement algorithms: A preference study," American Journal of Roentgenology, vol. 175, no. 1, pp. 45-51, 2000.

[8] C. Chang and A. Laine, "Enhancement of mammograms from oriented information," in Proc. International Conference on Image Processing, vol. 3, 1997, pp. 524-527.

[9] L. Gagnon, J. Lina, and B. Goulard, "Sharpening enhancement of digitized mammograms with complex symmetric daubechies wavelets," in Proc. IEEE 17th Annual Conference on Engineering in Medicine and Biology Society, 20-25 Sep., 1995, vol. 1, pp. 543-544.

[10] A. Papadopoulos, D. Fotiadis, and L. Costaridou, "Improvement of microcalcification cluster detection in mammography utilizing image enhancement techniques," Computers in Biology and Medicine, vol. 38, no. 10, pp. 1045-1055, 2008.

[11] J. Scharcanski and C. Jung, "Denoising and enhancing digital mammographic images for visual screening," Computerized Medical Imaging and Graphics, vol. 30, no. 4, pp. 243-254, 2006.

[12] G. Nason and B. Silverman, "The stationary wavelet transform and some statistical applications," Wavelets and Statistics, pp. 281-299. Springer, New York, 1995.

[13] A. Jumah, "Denoising of an image using discrete stationary wavelet transform and various thresholding techniques," Journal of Signal and Information Processing, vol. 4, no. 1, pp. 33-41, 2013.

[14] X. Qi, J. Tyler, and O. Pianykh, "Diagnostically lossless medical image compression via wavelet-based background noise removal," International Society for Optics and Photonics, AeroSense, pp. 470-480, 2000.

[15] A. K. Jain, Fundamentals of Digital Image Processing, Prentice Hall, 1989.

[16] T. Zaveri and M. Zaveri, "A novel region based multimodality image fusion method," Journal of Pattern Recognition Research, vol. 6, no. 2, pp. 140-153, 2011.

[17] S. Wu, S. Yu, Y. Yang, and Y. Xie, "Feature and contrast enhancement of mammographic image based on multiscale analysis and morphology," Computational and Mathematical Methods in Medicine, p. 8,2013 .

[18] S. Agaian, K. Lentz, and A. Grigoryan, "A new measure of image enhancement," in Proc. IASTED International Conference on Signal Processing \& Communication, 2000, pp. 19-22.

[19] R. Gonzalez and R. Woods, Digital Image Processing, Prentice Hall India, 2002.

[20] J. Suckling et al., "The mammographic image analysis society digital mammogram database," in Proc. 2nd International Workshop on Digital Mammography, Excerta Medica, Amsterdam, 1994, pp. 375-378. 
[21] K. K. Rajkumar and G. Raju, "Enhancement of mammograms using tophat filtering and wavelet decomposition," Journal of Computer and Mathematical Sciences, vol. 2, no. 6, pp. 780-898, 2011.

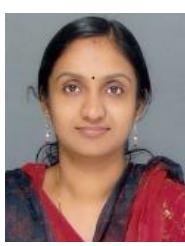

Arya Devi P. S. obtained her B.E degree in electronics and communication engineering from Bharatidasan University, received the M.Tech degree in applied electronics from Mahatma Gandhi University, Kerala in 2008. Her areas of interest include image compression, information theory and coding. She has memberships in societies like IEEE, ISTE and IETE.

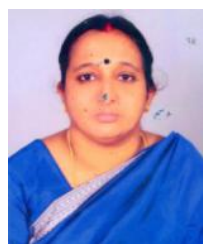

M. G. Mini has received her $\mathrm{PhD}$ degree from CUSAT, Kerala in 2005. Her areas of interest include digital image processing, VLSI, etc. She has several research papers published in national and international journals to her credit. 